\title{
XXVI. Bemerkungen zu der Compensationsmethode des Herrn A. E. Tutton und über die Verwendung von Quarz als Vergleichskörper bei dilatometrischen Messungen.
}

\author{
Von \\ C. Pulfrich in Jena. \\ (Hierzu 4 Textfiguren.)
}

Im 30. Bande dieser Zeitschrift (S. 529-567) beschreibt Herr Tutton unter wiederholtem Hinweis auf meinen in der Zeitschrift für Instrumentenkunde 1893, S. 365 veröffentlichten Aufsatz »Ueber das Abbe-Fizeau'sche Dilatometer $₫$ ein von den Herren Troughton and Simms in London hergestelltes sogenanntes Compensations-Interferenzdilatometer, welches auf der Anwendung des bekannten Fizeau'schen Interferenztischchens beruht und bei welchem die von Herrn Prof. Abbe angegebenen Verbesserungen der Fizeau-Benoit'schen Methode - Anwendung einer einzigen Marke, mikrometrisches Messverfahren und spectral zerlegtes Licht - zum Theil in der gleichen, zum Theil in einer mehr oder weniger abweichenden Form, wie bei dem von der Firma Carl Zeiss in Jena hergestellten Abbe-Fizeau'schen Dilatometer, in Anwendung gekommen sind.

Den specifischen Vorzug seines Apparates vor den bisherigen sieht Herr Tutton in der von ihm eingeführten sogenannten Compensationsmethode (vergl. weiter unten), von der er sagt, dass durch sie idie Empfindlichkeit der Interferenzmethode so bedeutend erhöht werde, dass es ganz unnüthig sei, eine Platte von grösserer Dicke als $5 \mathrm{~mm}$ anzuwenden *. Herr Tutton theilt mit, dass er den Apparat construirt habe, »um im Stande zu sein, die genaue Bestimmung der thermischen Ausdehnung von Krystallen künstlich dargestellter Substanzen auszuführen, weil es im Allgemeinen unmöglich sei, von solchen hinreichend grosse und noch homogene Krystalle darzustellen, um Platten von $10 \mathrm{~mm}$ oder mehr Dicke zu liefern, wie sie von den bisher beschriebe- 
nen $\Lambda$ pparatarten verlangt werden«. Bei seinem Apparate werde die Ausdehnung der drei Schrauben des Fizeau'schen Tischchens »compensirt und beseitigt, so dass die gesammte Ausdehnung der Substanz für die Messung benutzbar werde. Folglich sei es möglich, ein ebenso'genaues Resultat mit einem kleinen Krystalle zu erhalten, als vorher nur mit Hülfe eines viel grösseren Krystalles zu bekommen war \&. Die durch den Druck hervorgehobenen Satztheile sind von mir als solche ausgezeichnet worden.

Gegen diese Ausführungen des Herrn Tutton habe ich einzuwenden:

1) dass die bisherige Versuchsanordnung selbstverständlich auch bei Objecten von weniger als $10 \mathrm{~mm}$ Dicke angewandt werden kann und wiederholt angewandt worden ist und dass

2) durch die sogenannte Compensationsmethode, welche übrigens ohne Weiteres auch bei den bisherigen Apparaten sich verwenden lässt, die Genauigkeit der Messung nicht gesteigert, sondern vermindert wird.

Die Compensationsmethode des Herrn Tutton beruht auf der Anwendung einer Aluminiumplatte von bestimmter Dicke, welche auf oder unter die zu untersuchende Krystallplatte und mit dieser zusammen auf das von Herrn Tutton angewandte Tischchen aus Platin-Iridium gelegt wird. Aluminium hat einen Ausdehnungscoëfficienten $10^{-8} \cdot(2204+2,12 t)$, welcher sehr nahe gleich ist dem $2 \frac{1}{2}$ fachen des Ausdehnungscoëfficienten von PlatinIridium $\left.10^{-8} \cdot(860+0,456 t)^{1}\right)$. Für eine Aluminiumplatte, deren Dicke gleich ist (rund) $\frac{2}{5}$ der Länge der Schrauben, ist somit die Aenderung der Dicke gleich der Längenänderung der Schrauben. Die Ausdehnung der Schrauben ist also compensirt; das zu untersuchende Object - die Dicke desselben ist bis auf die Dicke der Luftschicht, an der die Interferenzstreifen beobachtet werden, gleich $\frac{3}{5}$ der Länge der Schrauben - steht gewissermaassen ausdehnungslosen Schrauben gegenüber. Natürlich kann die Compensation wegen des verschiedenartigen Ganges der Ausdehnung von Aluminium und Platin-Iridium keine vollkommene sein. Es bleibt, wie auch Herr Tutton hervorhebt, ein Compensationsrest bestehen, der in jedem einzelnen Falle nach Grösse und Vorzeichen genau zu bestimmen und in Anrechnung zu bringen ist. Hiervon abgesehen, tritt statt der Differenz der Ausdehnung von Object und Schrauben - wie es bei der bisherigen Versuchsanordnung der Fall war - die Ausdehnung des Objectes nahezu ungeändert in die Erscheinung.

Dieser Umstand, welcher für Demonstrationszwecke jedenfalls einige Beachtung verdienen dürfte, hat offenbar Herrn Tutton verleitet, für sein Verfahren eine grössere Genauigkeit zu postuliren. Es lässt sich sofort zeigen, dass Herr Tutton hier in einem Irrthume befangen ist.

1) Die beiden Werthe sind der Tutton'schen Arbeit entnommen, 
Bezeichnet man die Länge der Platin-Iridiumschrauben, in Millimetern gemessen, mit $E$, den linearen Ausdehnungscoëfficienten mit $\beta$, die entsprechenden Werthe für den Compensator mit $C$ und $\gamma$ und für den zu untersuchenden Körper mit $L$ und $\alpha$, so gelten für die Dicke $d$ der Luftschicht und deren Aenderung $\mathcal{A} d$ für eine Temperatursteigerung von $t_{1}$ auf $t_{2}$ die bekannten Beziehungen:

$$
\begin{aligned}
& d=E-C-L \\
& \Delta d=f \frac{\lambda}{2}=E \beta\left(t_{2}-t_{1}\right)-C \gamma\left(t_{2}-t_{1}\right)-L \alpha\left(t_{2}-t_{1}\right) .
\end{aligned}
$$

Hieraus erhält man die Ausdehnung des Objectes, gemessen in halben Wellenlängen, mit anderen Worten die Zahl $(F)$ der unter dem Einflusse der Ausdehnung des Objectes allein an der Marke vorüberziehenden Interferenzstreifen $\mathrm{zu}$ :

$$
\frac{L \alpha\left(t_{2}-t_{1}\right)}{\frac{\lambda}{2}}=F=\frac{E \beta\left(t_{2}-t_{1}\right)}{\frac{\lambda}{2}}-\frac{C \gamma\left(t_{2}-t_{1}\right)}{\frac{\lambda}{2}}-f .
$$

In diesem Ausdrucke sind die beiden ersten Glieder der rechten Seite gemäss der Tutton'schen Versuchsanordnung zusammen nahezu gleich Null, die Zahl $f$ ist nur sehr wenig verschieden von $F$.

In Bezug aber auf den Grad der Unsicherheit, mit welcher die beiden Werthe $f$ und $F$ behaftet sind, unterscheiden sich dieselben ganz erheblich von einander. Denn ausser dem bei der Bestimmung von $f$ gemachten Fehler, den wir in Anbetracht der Leistungsfähigkeit des mikrometrischen Messverfahrens gleich $\pm 0,01$ setzen wollen, kommen für die Beurtheilung der Unsicherheit von $F$ noch in Anrechnung die den beiden ersten Gliedern anhaftenden Fehlerwerthe. Denn jedes dieser beiden Glieder muss ebenso wie die Grösse $f$ durch eine besondere Versuchsreihe ermittelt, bezw. aus einer solchen Versuchsreihe durch Rechnung abgeleitet werden, wie dies auch Herr Tutton selbst init grösster Sorgfalt gethan hat ${ }^{1}$ ). Setzen wir für das erste Glied, unbekümmert um diejenige Unsicherheit, welche durch die relativ grosse Luftcorrection bedingt ist, den Fehler ebenfalls gleich $\pm 0,01$ und denjenigen des zweiten Gliedes gleich $\pm 0,02-$ die Unsicherheit des zweiten Gliedes ist doppelt so gross wie die des ersten Gliedes, weil die Messung der Ausdehnung des Compensators durch eine Differenzmessung erfolgt, die Unsicherheit des ersten Gliedes also auch in das zweite Glied mit eintritt -, so ergiebt sich die Unsicherheit des Werthes $F$ zu $\pm 0,04$.

Untersucht man dem gegenüber dasselbe object nach der früheren Versuchsanordnung ohne den Compensator, reducirt also die Länge der

1) Im Interesse der Genauigkeit wäre die directe Bestimmung der Differenz der beiden Glieder vortheilhafter gewesen. 
Schrauben auf den Betrag $E^{\prime}=L+d$, so erhält man für die Ausdehnung des Objectes, wiederum gemessen in halben Wellenlängen, den Werth:

$$
\frac{L \alpha\left(t_{2}-t_{1}\right)}{\frac{\lambda}{2}}=F=\frac{E^{\prime} \beta\left(t_{2}-t_{1}\right)}{\frac{\lambda}{2}}-f^{\prime},
$$

mit einer Unsicherheit von $F$ im Betrage von $\pm 0,02$, wobei für jeden der beiden rechts stehenden Summanden die gleiche Unsicherheit $( \pm 0,01)$ angenommen ist, wie für die entsprechenden Grössen des Ausdruckes $A$.

Der Umstand, dass die Zahl $f$ des Ausdruckes (A) grösser ist als die Zahl $f^{\prime}$ des Ausdruckes (B), die Zahl $f$ also mit einem kleineren procentualen Fehler behaftet ist als die Zahl $f^{\prime}$, ist für die Beurtheilung des der Zahl $F$ anhaftenden Fehlers gegenstandslos. Denn es kommen hierfür nicht die procentualen, sondern die absoluten Fehler der einzelnen Glieder in Anrechnung.

Hieraus geht hervor, dass der gefundene Werth für den Ausdehnungscoëfficienten $\alpha$ mit einem Fehler

$$
d \alpha=\frac{\frac{\lambda}{2}}{L\left(t_{2}-t_{1}\right)} d F=\frac{\alpha \cdot d F}{F}
$$

behaftet ist, welcher bei Anwendung der Tutton'schen Compensationsmethode unter sonst gleichen Bedingungen mindestens doppelt so gross ist, als bei der bisherigen Versuchsanordnung ohne den Compensator.

Ich sehe daher in der Anwendung der Compensationsmethode, insoweit es sich um das Bestreben handelt, eine grössere Genauigkeit zu erzielen, nicht nur eine unnöthige Complication für das Beobachtungsverfahren, sondern auch eine directe Schädigung der Genauigkeit der Messung. Gegen die Anwendung der Compensationsmethode für die Untersuchung solcher Körper, welche sich nicht oder nur schwer mit einer spiegelnden Planfläche versehen lassen, habe ich kaum etwas einzuwenden. Denn ich habe selbst schon in meiner früheren Arbeit für derartige Fälle die Anwendung einer Hülfsplatte aus Quarz oder Glas empfohlen. Selbstverständlich kann ich aus den vorstehend angeführten Gründen der Anwendung einer Aluminiumplatte nicht den Vorzug zugestehen, welchen Herr Tutton für sie hinsichtlich der Genauigkeit der Messung beansprucht. Auch habe ich einiges Bedenken gegen die Benutzung von Aluminium als Vergleichskörper wegen der relativ geringen Widerstandsfähigkeit des Materials gegen äussere Angriffe.

Die vorstehenden Bemerkungen veranlassen mich, im Folgenden den Lesern dieser Zeitschrift über einige neuere in Jena ausgeführte dilatometrische Untersuchungen, insbesondere über 


\section{eine neue Versuchsanordnung mit Quarz als Vergleichskörper}

$\mathrm{zu}$ berichten. Zum Theil finden sich die Ergebnisse dieser Untersuchungen niedergelegt in einer Arbeit des Herrn E. Reimerdes, »Untersuchungen über die Ausdehnung des Quarzes durch die Wärme«, Inauguraldissertation Jena 1896, und in einer vor Kurzem von mir veröffentlichten Arbeit »Ueber einen Interferenzmessapparat « 1 ).

Bei meinen Arbeiten mit dem Abbe-Fizeau'schen Dilatometer ${ }^{2)}$ hatte sich als ein Nachtheil des von mir benutzten und in den beiden nachstehenden Figg. 1 und 2 in natürlicher Grösse abgebildeten. Stahltischchens ergeben, dass die drei Stahlschrauben, obschon sie hinter einander

Fig, 1,

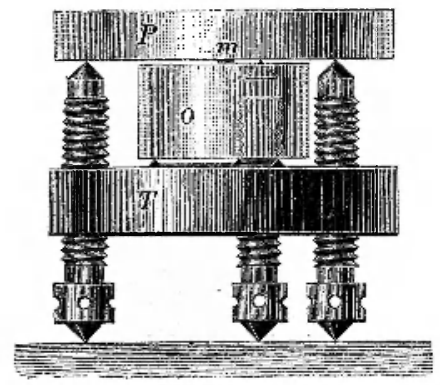

Fig. 2.

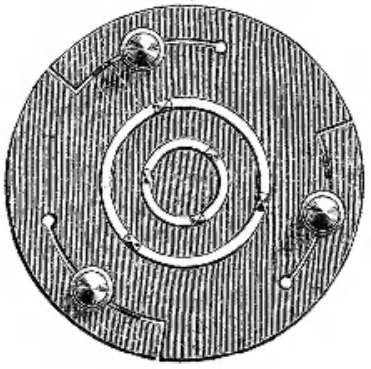

von einer Stahlstange abgeschnitten waren und durch Ausglühen und $\mathrm{Ab}$ kühlen die gleiche Behandlung erfahren hatten, keine genau übereinstimmende Ausdehnung besassen und ausserdem nicht vollkommen frei waren von thermischer Nachwirkung (man vergl. des Näheren meinen Aufsatz über das Dilatometer). Für dilatometrische Messungen, bei welchen es auf den erreichbaren höchsten Grad der Genauigkeit ankommt, schien mir daher das Stahltischchen trotz seiner sonstigen Vorzüge nicht mehr geeignet und ich bin zu der im Folgenden beschriebenen Versuchsanordnung mit Quarz als Vergleichskörper übergegangen.

In der nebenstehenden Fig. 3 ist die in der Zeiss'schen Werkstätte hergestellte Quarzröhre, welche als Ersatz für das Stahltischchen dienen soll, in natürlicher Grösse abgebildet. Die Röhre hat einen inneren Durchmesser von etwa $25 \mathrm{~mm}$ und eine Wandstärke von $5 \mathrm{~mm}$. Sie wurde aus einer senkrecht zur optischen Axe geschliffenen ca. $10 \mathrm{~mm}$ dicken und von sehr nahe parallelen, ebenen Flächen begrenzten Quarzplatte durch Aus-

1) Pulfrich, Zeitschr. f. Instrumentenkunde 1898, 18, 261.

2) Siehe ferner Pulfrich, Wiedem. Ann. 1892, 45, 609 und O. Schott, Ueber die Ausdehnung von Gläsern und über Verbundglas, Verhandlungen des Vereins zur Beförderung des Gewerbefleisses. Berlin 1892. 
bohren erhalten. Jedes der heiden Rohrenden ist bis auf drei kleine vorspringende Flächenstücke ausgearbeitet. Um eine vollkommen sichere Berührung dieser Füsschen mit der Bodenplatte und mit der Deckglasplatte zu erzielen, empfiehlt es sich, vor der Benutzung der Röhre die vorgenannten Flächenstückchen matt zu schleifen.

Die Quarzröhre ist, was die Zuverlässigkeit ihrer Angaben anbetriflt, dem Stahltischchen entschieden vorzuziehen. Denn Quarz ist ein Körper, welcher in der Natur stets in der gleichen Beschaffenheit vorkommt, meines Wissens frei ist von thermischer Nachwirkung ${ }^{1}$ ) und hinsichtlich seiner Homogenität - es kommt für unsere Zwecke nur homogenes, von Verwachsungen und Zwillingsbildungen vollkommen freies Material in Frage - einer vorherigen optischen Prüfung unterworfen werden kann. Die Richtung der optischen Axe, deren Anwendung aus Gründen der Symmetrie sich empfiehlt, kann ebenfalls auf optischem Wege nach bekannten Methoden und in prak-

Fig. 3.

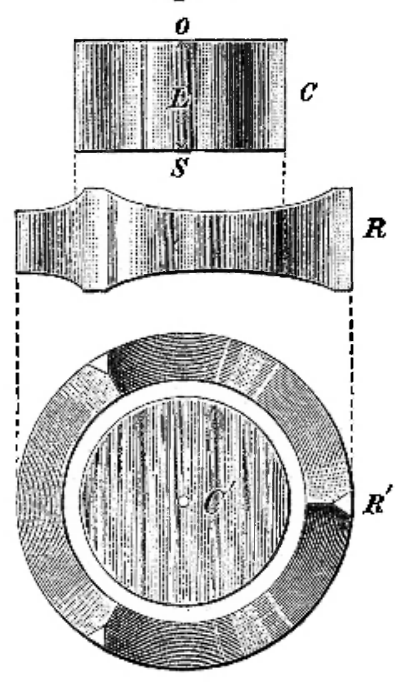
tisch ausreichender Weise festgelegt werden.

Die Handhabung der Quarzröhre ist natürlich nicht so bequem wie die des Stahltischchens, da das Hülfsmittel, durch Verstellen der drei Schrauben die Länge derselben der Höhe des Objectes anzupassen, hier vollständig fehlt. Aber deshalb ist das Verfahren noch nicht gerade ein umständliches oder besonders schwieriges zu nennen. Nach den Untersuchungen des Herrn Reimerdes (l. c.), bei welchen das Verfahren zum ersten Male verwerthet wurde, ist dasselbe als praktisch durchaus erprobt anzusehen ${ }^{2}$. Auch ist es schon von verschiedenen anderen wissenschaftlichen Instituten in Benutzung genommen worden.

In der Hauptsache enthält die Reimerdes'sche Arbeit die Bestimmung des Ausdehnungscoefficienten von Quarz in der Richtung der optischen Axe für die Temperaturen zwischen $5^{0}$ und $230^{\circ} \mathrm{C}$. Die Versuche wurden mit Hülfe des Abbe'schen Dilatometers und unter Benutzung des demselben beigegebenen Heizapparates (Verwendung von Leinöl als Heizflüssigkeit) ausgeführt.

1) Ueber die von mir an optischen Gläsern beobachteten Erscheinungen thermischer Nachwirkung siehe den oben erwähnten Aulsatz des Herrn Dr. O. Schott.

2) In gleicher Weise wurde die Quarzröhre benutzt beì den Messungen des Herrn Kellner (Jena) an Turmalin, mitgetheilt durch Herrn Prof. W. Voigt, Nachrichten d, k, Gesellsch, d. Wissensch, zu Göttingen, mathem.-physik. Klasse 1898, Hệt 2. 
Die Bestimmung des Ausdehnungscoëfficienten erfolgte an der Quarzröhre nach dem in nebenstehender Fig. 4 abgebildeten Versuchsarrangement. Die Röhre wurde auf die ebene Fläche einer Bodenplatte ( $G$ in Fig. 4)

Fig. 4.

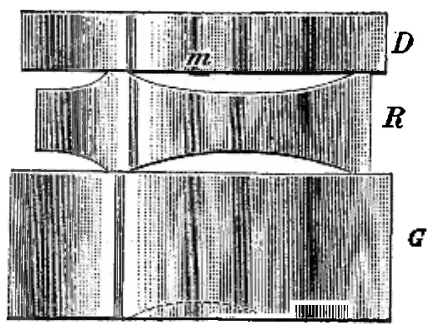
gestellt und oben die bekannte keilförmige Deckglasplatte aufgelegt. In Folge der vorhandenen geringen Neigung der beiden Rohrenden $\mathrm{zu}$ einander waren die Interferenzstreifen an der ca. $10 \mathrm{~mm}$ dicken Luftschicht ohne weitere Bearbeitung eines der drei Füsschen beobachtbar.

Als Ergebniss dieser Messungen giebt Reimerdes den axialen $\Lambda$ usdehnungscoefficienten von Quarz an zu:

$$
\beta_{t}=10^{-8} \cdot(692,5+1,689 t) \text {, }
$$

gültig für das Temperaturintervall von $5^{0}$ bis $230^{\circ} \mathrm{C}$.

Verglichen mit dem von Herrn Benoit (1888) bestimmten Werthe $10^{-8} \cdot(711,1+1,712 t)$ zeigt der vorstehende, wenigstens was das erste (constante) Glied anbetrifft, nicht unerhebliche Abweichungen, welche eine vollständige Erklärung zur Zeit noch nicht gefunden haben (man vergl. die diesbezügliche Discussion in der Reimerdes'schen Arbeit). In Anbetracht der Wichtigkeit einer genauen Kenntniss des Ausdehnungscoefficienten von Quarz für unsere Zwecke werden gegenwärtig im physikalischen Laboratorium der Universität Jena neue Bestimmungen üher die Ausdehnung von Quarz in den beiden Hauptrichtungen und untèr etwas anderen Versuchsbedingungen (Verwendung des Interferenzmessapparates und des weiter unten èrwähntèn Heizkörpers) vorgenommen. Auch wèrden diesè Untersuchungen auf Temperaturen bis $400^{\circ}$ und mehr ausgedehnt.

Es war von Interesse zu sehen, zu welchem Werthe man für den Ausdehnungscoefficienten der Schrauben des von mir benutzten Stahltischchens gelangt, wenn man den obigen Werth für Quarz zu Grunde legt. Herr Reimerdes hat diese Messungen ausgeführt und unter Benutzung des bei der Herstellung der Quarzröhre erhaltenen Vollcylinders ( $C$ in Fig. 3) den gesuchten Werth ermittelt $\mathrm{zu} 10^{-8} \cdot(1011+0,89 t)$. Dieser Werth stimmt mit dem von mir benutzten $\left.{ }^{1}\right) 10^{-8} \cdot(1033+0,60 t)$ für eine Mittètemperatur von ètwá $55^{\circ} \mathrm{C}$. $\left(15^{0}-95^{\circ} \mathrm{C}\right.$.), auf welche sich meine sämmtlichen früheren Messungen an Gläsern etc. beziehen, bis auf Abweichungen von weniger als $1 \%$ überein. Für den Vergleich jener Messungsresultate unter einander haben diese Abweichungen keine Bedeutung.

Die Ergebnisse der von Herrn Reimerdes vorgenommenen Untersuchung des Quarzcylinders hinsichtlich des Einflusses der Temperatur auf die Lichtbrechung von

1) Benoit giebt für Stahl an $10^{-8} \cdot(1033+1,20 t)$. 
Quarz in der Richtung der optischen Axe sind folgende. Die Untersuchungsmethode ist dieselbe wie bei Fizeau: man beobachtet die Interferenzstreifen, welche an der schwach keilförmigen Quarzplatte durch Reflexion an den beiden Endflächen entstehen, und bestimmt ihre Lagenänderung für eine bestimmte Temperaturdifferenz. Derjenige Theil der Verschiebung, welcher von der Ausdehnung des Quarzcylinders herrïhrt und welcher durch die Messungen an der Quarzröhre bekannt ist, wird in Abrechnung gebracht. Der Rest giebt dann das Maass für die Aenderung des Brechungsindex in Folge der Temperaturänderung. Auf diese Weise erhielt Herr Reimerdes aus zehn zwischen den Temperaturen $20^{\circ}$ und $230^{\circ} \mathrm{C}$. gelegenen Versuchsreihen den nachstehenden Werth für die Aenderung des Brechungsindex (ord. Strahl) von Quarz und gültig für das Licht der grünen Quecksilberlinie:

$$
\Delta \mathfrak{R}_{t}=-10^{-5} \cdot\left(0,5882-0,000272 t+0,000005327 t^{2}\right) .
$$

Dieser Ausdruck besagt, dass der Brechungsindex von Quarz eine mit wachsender Temperatur beschleunigte Abnahme erfährt. Die von mir früher nach der prismatischen Methode (siehe Wiedem. Ann. 45, 609; Ref. diese Zeitschr. 23, 625) bestimmten Zahlenwerthe sind mit den obigen in guter Uebereinstimmung.

Endlich ist die Quarzröhre von Herrn Reimerdes zur Bestimmung des Ausdehnungscoëfficienten einiger Gläser des Jenaer Glaswerkes Schott und Gen. benutzt worden. Ich beschränke mich hier auf die Skizzirung der Versuchsanordnung.

Das zu untersuchende Object erhält eine Dicke, welche nur wenige Hundertel Millimeter kleiner ist als die Höhe der Quarzröhre. Die der planen Fläche gegenüberliegende Fläche wird bis auf drei vorspringende Punkte ausgearbeitet. Mit diesen Punkten kommt dann das Object innerhalb der Quarzröhre auf die Bodenplatte zu stehen. Ueber die Quarzröhre kommt wieder die keilförmige Deckglasplatte zu liegen. Die Regulirung des Abstandes der Interferenzstreifen wird durch vorsichtiges Nachschleifen eines der Fusspunkte des Objectes, bezw., wenn nur eine geringe Aenderung gewünscht wird, durch Drehen der Quarzröhre um die Rohraxe bewerkstelligt.

Die Quarzröhre lässt sich auch zur Untersuchung solcher Objecte verwenden, deren Dicke wesentlich kleiner ist als die Länge der Quarzröhre. Es ist dann nur nöthig, die Dickendifferenz - bis auf einen kleinen Bruchtheil von wenigen Hundertel Millimetern für die wirksame Luftschicht durch eine senkrecht zur optischen Axe geschliffene und auf der einen Seite mit drei Füsschen versehene Quarzplatte auszugleichen. Die hierbei in Anrechnung zu bringende wirksame Länge der Quarzröhre ist gleich der Differenz der Rohrlänge und der Plattendicke.

Selbstverständlich können solche Quarzplatten, von denen jedesmal mehrere (von verschiedener Dicke) einer Quarzröhre beigegeben werden, auch für sich als Vergleichskörper für die Untersuchung von Glas- oder Metallröhren benutzt werden. - 
Endlich gebe ich im Folgenden, zur Vervollständigung des Vorhergehenden, ein Referat über den oben erwähnten Aufsatz »Ueber einen Interferenzmessapparat , sowie eine kurze Beschreibung der Versuchsanordnung für die Verwendung dieses Apparates zu dilatometrischen Messungen in Verbindung mit dem neuen, bereits oben kurz erwähnten Heizkörper.

Mit der Construction des Interferenzmessapparates habe ich den Zweck verfolgt, die bekannten Vortheile des Abbe'schen Dilatometers, welche in der Anwendung monochromatischen Lichtes verschiedener Wellenlänge und in der mikrometrischen Ausmessung der Interferenzstreifen begründet sind, auch anderweitigen Aufgaben, bei denen es ebenfalls auf die Beobachtung und die Ausmessung von Interferenzstreifen ankommt, zugänglich zu machen.

Daher wurden im Wesentlichen die zur Beobachtung und Ausmessung der Interferenzstreifen dienenden Einrichtungen des Abbe'schen Dilatometers beibehalten. Im Uebrigen aber habe ich den Apparat von den den speciellen Zwecken des Dilatometers dienenden Apparattheilen losgelöst und mit verschiedenen Neueinrichtungen ausgerüstet, durch welche in erster Linie erreicht worden ist, dass man den Apparat auf jedes beliebige Object, an dem die Interferenzerscheinung beobachtet werden soll, einstellen kann, und durch welche ausserdem die Möglichkeit geboten ist, dass man die Interferenzerscheinung sowohl im monochromatischen (spectralzerlegten), als auch im weissen Lichte beobachten kann. Die hierzu dienenden Hülfseinrichtungen bestehen erstens in einem 900-igen Reflexionsprisma, welches an Stelle der beiden Prismen $P_{1}$ und $P_{2}$ des Dilatometers vor dem Fernrohrobjectiv so befestigt ist, dass man es um die Fernrohraxe drehen und auch von dem Apparate herunternehmen kann, und zweitens in einem geradsichtigen Amiciprisma, welches nach Bedarf unmittelbar vor dem Auge des Beobachters in das Beobachtungsrohr eingesteckt wird.

Endlich ist durch Einfügung eines um die Fernrohraxe drehbaren Dove'schen Reflexionsprismas in den Strahlengang eine besondere Vorkehrung dafür getroffen, dass man die im Gesichtsfelde des Fernrohres zur Abbildung gelangenden Interferenzstreifen richten, d. h. den Messfäden des Mikrometers parallel einstellen kann, ohne dass man am Objecte dieses Ausrichten der Streifen vorzunehmen braucht, welches in vielen Fällen von vornherein unmöglich ist.

Wegen der Einzelheiten der Construction muss auf die Arbeit selbst verwiesen werden, ebenso wegen der Verwendbarkeit des Apparates zu den verschiedenen dort angegebenen Interferenzversuchen.

Ueber die Construction des neuen Heizkörpers ist bereits an anderer Stelle eingehend berichtet worden. Er findet sich ausführlich beschrieben und abgebildet in einer Arbeit des Herrn Prof. J. O. Reed, 
»Ueber den Einfluss der Temperatur auf die Lichtbrechung von Gläsern etc. ${ }^{1}$ ). « Mit Hülfe des ursprünglich nur für diese Zwecke construirten Apparates ist es möglich gewesen, die Bestimmung der Lichtbrechung von Gläsern etc. bei Temperaturen, welche in der Nähe der Erweichungstemperatur des Glases $\left(400^{\circ}-450^{\circ} \mathrm{C}\right.$.) gelegen sind, fast ebenso sicher und bequem vorzunehmen, wie bei gewöhnlicher Temperatur. Die Resultate lassen erwarten, dass der Heizapparat auch bei dilatometrischen Messungen gute Dienste leisten wird.

Der Ileizkörper besteht in der Hauptsache in einer ca. $50 \mathrm{~kg}$ schweren, durch Gasflammen erwärmten Kupfermasse, in deren Inneren das zu untersuchende Object ohne Berührung mit der Metallmasse aufgestellt ist. Zu dem Ende ist der Heizkörper, ein oben abgerundeter Cylinder, mit einer verticalen cylindrischen Durchbohrung versehen, welche von oben durch einen massiven Metallconus mit darin eingesetztem Thermometer verschlossen ist. Von unten ragt in den Hohlraum ohne Berührung mit dem eigentlichen Heizkörper eine verticale Drehungsaxe, auf welche das zu untersuchende Object zu stehen kommt. Um eine directe Berührung des Objectes mit der Metallmasse der. Axe zu vermeiden, ist hier eine Glasröhre mit aufgelegtem Deckel eingeschaltet.

Der Heizkörper wird von einem kräftigen Träger mit drei Armen in bestimmter Höhe festgehalten.

Zum Einblick in den inneren Hohlraum ist die Kupfermasse in der Höhe des Objectes bis zur Mitte cylindrisch durchbohrt. Die Oefinung wird durch eine beiderseits mit planparallelen Glasplatten verschlossene Metallrühre geschlossen. Letztere, sowie die ganze Kupfermasse, sind nach aussen mit Asbestpappe bedeckt.

Entsprechend diesen Versuchsbedingungen wird an Stelle der keilförmigen Deckglasplatte ein mit der Einstellungsmarke versehenes 90"-iges Reflexionsprisma auf die Quarzröhre gelegt und die gleichmässige Belastung der drei Füsse durch ein dem Glasprisma angehängtes Aluminiumprisma erzielt.

Die Erwärmung wird durch Gasflammen unter Anwendung von Gasdruckregulatoren bewerkstelligt. Die Brenner werden so unter den Heizkörper gestellt, dass die Flammengase denselben in einer auf der unteren Seite angebrachten Eindrehung berühren, von wo aus sie durch zehn gerade Schornsteine durch die Kupfermasse nach oben abziehen. Der Abzug kann durch. Drehen einer Scheibe am oberen Ende der Oeffnungen nach Belieben regulirt werden.

1) J. O. Reed, Inauguraldissertation Jena 1897; siehe auch Wiedem. Ann. 1898, 65, 707 . 
382 C. Pulfrich. Bemerk. zu der Compensationsmeth. des Hrn. A. E. Tutton etc.

Zum Heben und Senken des Heizkörpers dient ein an dem oberen Ende befestigtes Seil, welches über zwei an der Decke des Zimmers befestigte Rollen läuft und an dem anderen Ende ein Gegengewicht trägt. Nach erfolgtem Zusammensetzen des Interferenzapparates und der Einstellung des Beobachtungsapparates auf diesen wird der Heizkörper vorsichtig herabgelassen. Um hierbei jede Berührung des Heizkörpers mit dem Interferenztischchen zu vermeiden, wird vor dem Herablassen des Heizkörpers das Object durch eine auf die Axe gesteckte Röhre geschützt, welche dann später wieder durch Ausziehen nach oben entfernt wird.

Ueber die Versuchsergebnisse wird später geeigneten Ortes näher berichtet werden.

Jena, in Februar 1899. 